Journal of Social Sciences 8 (2): 196-201, 2012

ISSN 1549-3652

(C) 2012 Science Publications

\title{
Autism Worldwide: Prevalence, Perceptions, Acceptance, Action
}

\author{
Patricia B. Kopetz and E. Desmond Lee Endowed \\ Department of Education of Children with Disabilities, \\ Center for the Research and Study of Disability, \\ Education and Culture (CRSDEC), University of Missouri-St, Louis
}

\begin{abstract}
This metanalysis scrutinizes the growth of the neurodevelopment condition known as "autism" as a modern-day phenomenon. Over the past decade and worldwide, estimates of increases between $50 \%$ to over $2000 \%$ in cases of Autistic Disorder diagnoses have been charted, studied and discussed. The rise in diagnoses of autism impacts us all. The world seeks greater awareness and understanding of the condition's complexities; endeavors to provide research-proven strategies and accommodations to support affected individuals and their families; espouses evidence-based, best practices that adjust classroom pedagogy and teacher preparation methodology that best orient not only special education teachers, but ALL educators and related services personnel who communicate, collaborate and serve in the day-to-day, inclusive educational environment; and pursues responses from national and state education departments and ministries regarding their responsibilities to protect the civil rights of individuals with autism and improve the "systems" in which the children interact. Conclusions highlight the scramble to learn the researched and proven-successful information and productive therapies meaningful to students with autism and their families. Educators must learn and implement the interventions that enable individuals with autism to best cope, thrive and excel in environments that are safe and accepting of diversity. Recommended is continued research and creative programs that nurture and encourage the children with autism to achieve their potential.
\end{abstract}

Key words: Autism Awareness Care and Training Centre (AACT), Autism Spectrum Disorder (ASD), Society for Rehabilitation of Mentally Challenged (SOREM), State Diagnostic and Counseling Center (SDCC), Center for the Research and Study of Disability, Education and Culture (CRSDEC)

\section{INTRODUCTION}

Autism is a global health crisis that knows no borders -- it does not discriminate based on nationality, ethnicity or social status. It's high time that the world begins to recognize the scope of this problem and acts internationally and locally to improve the lives of the growing number of individuals and families affected by this devastating disorder."

Suzanne Wright, of Autism Speaks: Approximately 67 million people are affected by autism around the world and we believe the solutions will come from a community of science and research without boundaries (as) an important path to investigate causes, prevention, treatments and a cure for autism; increase international awareness of autism spectrum disorders; and advocate for the needs of all individuals with autism and their families in our global community.
Bob Wright, of Autism Speaks (Autism Speaks, 2008): Growth of the neurodevelopmental condition of autism is indeed a phenomenon (Posserud et al., 2006). Over the past decade and worldwide, estimates of increases between $50 \%$ to over $2000 \%$ in cases of Autistic Disorder diagnoses have been charted, studied and discussed (Centers for Disease Control and Prevention, 2011) Autism Society, 2010. The rise in diagnoses of autism impacts us all, as we search for greater awareness and understanding of the condition's complexities; endeavor to provide research-proven strategies and accommodations in direct support of affected individuals and their families; determine evidence-based, best practices that adjust classroom pedagogy and teacher preparation methodology that orients not only special education teachers, but ALL

Corresponding Author: Patricia B. Kopetz, Department of Education of Children with Disabilities,

Center for the Research and Study of Disability, Education and Culture (CRSDEC), University of Missouri-St, Louis 
educators and related services personnel who communicate, collaborate and serve in the day-to-day, inclusive educational environment; and pursue responses from national and state educational departments and ministries regarding their responsibilities to protect the civil rights of individuals with autism, improve the "systems" in which the children interact and prepare a more-aware and sensitive populous who strive to better understand and accept the characteristics commonly demonstrated by individuals with Autistic Disorder.

In countries throughout the world, scientists and researchers scramble to draw educators, physicians, product manufacturers and related specialists of many global industries to the need for researched and provensuccessful curricula, therapies, specialized devices and related supports necessary to guide, encourage and meaningfully educate in schools students with autism.

Prevalence rates of autism: In the United States of America, the Centers for Disease Control and Prevention, or CDC, conducts year-round studies that relate to and indicate the incidence and prevalence of autism diagnoses, also providing systematic public health monitoring of a wide range of diagnosed disorders. Their research is held to the highest standards of validity and reliability. The CDC defines persons with Autism Spectrum Disorder (ASD) as those who demonstrate "impairments in social, communicative and behavior development that typically are present before age 3 years and that often are accompanied by abnormalities in cognitive functioning, learning, attention and sensory processing" (Centers for Disease Control and Prevention, 2011). Further descriptive, Marchant et al. (2006), identify autism as "an impairment of social relationships, rigidity and inflexibility of thought processes and specific language impairment," and all three are present to render the diagnosis of autism (p. 230). They cite five major characteristics of autism: social withdrawal; impairment of language and communication; ritualistic, compulsive and repetitive behavior; demands for sameness or resistance to change; and abnormal responses to sensory events, or "stimulus over-selectivity." Diagnosing autism is much more complex than the description of those particular characteristics suggests (Marchant et al., 2006). Individuals with autism demonstrate degrees of impairment that may differ widely (Cohen, 1997).

Worldwide, population-based studies conducted before 1985 identified the prevalence of autism and related conditions among children under 18 years old to be approximately .5 per 1,000 children. The CDC's most recent studies suggest that children meeting the "Autistic Disorder" criteria range in numbers up to 12 per 1,000 children, worldwide. In the USA, rates of autism climbed "greater than tenfold" from rates identified during the 1980s, to a current estimate of 1 in 110 children (1\%) diagnosed with autism. Beginning in the 1990s, reported increases in autism diagnoses elevated public concern regarding the rise in prevalence of such behaviorally-defined conditions in children (Autism Speaks, 2010b) Centers for Disease Control and Prevention, 2009. Puzzling and confirmed through research, are the facts that there are no genetic or biologic markers for early and consistent identification of autism and there is no cure for the disability Centers for Disease Control and Prevention, 2009 Autism Society, 2010. Current estimates of the lifetime costs to care for a child with autism ranges from $\$ 3.5$ million to $\$ 5$ million. To accommodate the complex needs of families with members affected by autism, the United States incurs estimated costs that total nearly $\$ 90$ billion annually Autism Society, 2010.

Tracking the support given individuals with autism in the U.S.A. and its outlying areas, provides charted data that indicate a $2039 \%$ increase in autism from 1993-2008 (Fig. 1). Reports from 1993 identified that a total of 19,058 children (6-22 years of age) were supported for autistic disorders and in 2008, the number of children supported soared to 380,438 .

In the United Kingdom, autism spectrum disorders are recognized to occur in up to $1 \%$ of their population. That information alarming to most, the country views this knowledge as a major public health concern due, in part, to the early onset of autism, its lifelong persistence and the high levels of associated conditions. Researchers admit that little is known about the associated psychiatric disorders that may contribute to impairment (National Autistic Society, 2006; Simonoff et al., 2008).

Autism is a condition affecting populations worldwide. Prevalence rates per country can be difficult to determine. Posserud et al. (2010), the Autism Society 2010, Autism Speaks 2008 and (Wong, 2007) calculated and identified respective country's approximate prevalence rates of autism diagnoses of children living in other countries throughout the world and combined as follows:

- Australia: 6.25 in 1000

- China: 1.1 in 1000

- Denmark: Nearly 9 in 1000

- India: 1 in 250

- Japan: Nearly 3 in 1000

- Mexico: 2 to 6 per 1000

- Canada: 1 in 154

- Sweden: 1 in 188

- Finland: 1 in 833

- Denmark: 1 in 833

- Iceland: 1 in 769

- Philippines: 500,000 children, total

- Thailand: 180,000 children, total 


\section{J. Social Sci., 8 (2): 196-201, 2012}

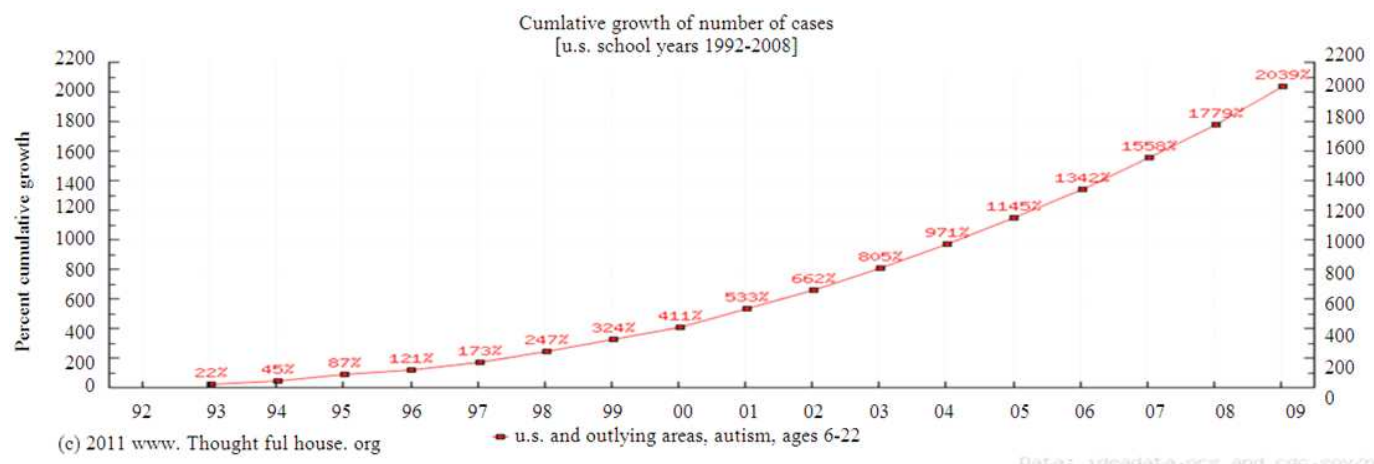

Fig. 1: The Growth of Autistic Disorder Diagnoses in the U.S.A.

Achieving accurate prevalence rates of autism is challenging, as autism can occur along with other disabilities.

Its definitions have been designed broader and more inclusive, such that diagnosing the disorder has changed over time. Diagnostic criteria for Autistic Disorder relies on behavioral observations of development that may preclude objectivity and there exist few, if any, population-based systems that tract the full range of autism over time National Institutes of Mental Health, 2011. Interestingly, autistic features in children are much more common than acknowledged by those in the medical community (Posserud et al., 2006). Speculations attempt to explain the reason for the staggering upsurge in diagnoses of autism among children, such as: differences of, or changes in, eligibility criteria; increased awareness; subjective judgments in the assessment process; non-response bias; changes in service availability; varied casefinding methods; and related issues of study design (Marchant et al., 2006; Posserud et al., 2010) Centers for Disease Control, 2009.

\section{Special projects for individuals with autism and their families:}

India-Ghana-Iceland: The Global Autism Project, for example, a nonprofit organization dedicated to providing resources, awareness and greater understanding of autism, is on a quest to provide services to individuals with autism in underserved communities throughout the world. Project programs challenge communities on respective continents to educate their citizenry through promoting acceptance and compassion, education and outreach and training for persons with autism. The Project touts a "community empowerment model" that supports systemic change, providing knowledge through workshops and hands-on training. Clinical services provided are supervised by Board Certified Behavior Analysts. Its unique approaches are supported with the goal of achieving for individuals with autism total access to effective services Global Autism Project, 2011.

Likewise, the Autism Awareness Care and Training Centre (AACT) of Accra, Ghana, helps parents of children with autism improve their understanding of the disability and provide them a safe, supportive place for help. A key goal of AACT's mission is for children with autism "to function more effectively in society." In their country, as is the case with many worldwide, lack of public information or knowledge about autism results in the families feeling ostracized from their communities and extended families. It is not uncommon for Ghanans to perceive mothers of children with autism as "witches," whose children possess evil spirits-resulting in families who experience shame, pain and trauma due to their children's characteristics of the disability. Through print, television and radio media outlets, AACT sponsors public awareness campaigns; provides lifeskills training; teaches language and communication skills, art classes and physical education; executes field trips and extra-curricular activities; and arranges respite care for the affected families.

In Chandigar, India, the Society for Rehabilitation of Mentally Challenged (SOREM) provides programs that foster "societal interaction," and they regard physical fitness as critical for individuals with autism. Program leaders design "functional education" approaches suited to meet each individual's special needs. Services provided include physiotherapy and speech and occupational therapies; music and dance; indoor and outdoor sports activities; specialized fitness methods by way of yoga, pranayama and meditation; and focused, vocational endeavors, like weaving, block printing and candle-making Society for Rehabilitation of the Mentally Challenged, 2011.

In Iceland, the Directorate of Health reports that at least $99 \%$ of children are served by fifty-five primary 


\section{J. Social Sci., 8 (2): 196-201, 2012}

health care centers and every child is examined by a physician at ages 6-12. Such a comprehensive system of health care is designed to determine medical and developmental disorders of children at their youngest ages. Children who demonstrate characteristics that may align with autism are referred for health and/or educational services provided by the State Diagnostic and Counseling Center (SDCC), which is supervised by developmental pediatricians and those of related health professions, such as: psychologists, physiotherapists, occupational therapists, social workers, special education teachers and speech and language pathologists. Any professional who suspects that a child has a serious developmental disorder, like autism, is required by law to refer the child to the SDCC. Although efficient and all-embracing, Iceland's health care system faces increased numbers of individuals with autism, such that the system cannot cope with the demand for treatment (Magnusson and Saemundsen, 2001).

The need for pedagogy that welcomes students with autism: There is worldwide concern regarding the "inclusiveness" of children with autism in schools and in classrooms that should comfortably accommodate children with wide-ranging disabilities that include autism. A critical concern of teachers responsible for students' learning in the inclusive classroom, is their lack of knowledge and understanding of, as well as professional preparation for, accommodating the characteristics and needs of students on the autism spectrum (Bellini et al., 2011). All teachers should be prepared to recognize and honor the legal and civil rights that protect all students and especially those with disabilities. A strong-quality, teacher preparation program must also address a host of significant factors, that not only include knowledge of the diverse experiences that children bring with them to school and the children's wide range of languages, cultures and exceptionalities (like autism), but teachers must also possess a learning repertoire of contemporary and bestpractice instructional strategies that indeed meet the individual learning styles of the children they serve. As they juggle varied subject matter simultaneously, effective teachers monitor each student's needs and responses, while seizing "teachable moments." They transition their students and responsibilities smoothly among various schoolday activities, while managing time, materials and equipment. Adding to daily rigor the planning of specific programs and strategies that assist students with autism to remain on task and to focus on important stimuli and cues, so that students gain the most from inclusive instruction-and therein lies the interesting, pedagogical challenge (Mehring and Dow, 2001).
Making their case for promoting the "core curriculum" as the classroom-based, instructional content and goals provided students of all ability levels, Kurth and Mastergeorge (2010) found in their study that, when taught in the inclusive setting, adolescents with autism achieved significantly higher scores on competency exams (i.e., achievement tests) than peers with autism who were instructed in segregated, selfcontained classroom settings (p. 8). Placed in the inclusive classroom environment, students with autism proved to experience heightened levels of social interaction and opportunities for skill development in academic-based areas of study. They actively engaged communication skills among students of all ability levels. Their areas of academic strength were typically found in concrete, procedural tasks. Because too often, rather than challenged by core curriculum expectations, students with autism learn from curricula that focuses heavily on activities, functional skills and/or specific, individualized program goals that are known to lack cohesion or continuity, students with autism miss out on the scope-and-sequence of academic skill attainment and progress. Meaningful, academic benefit for students with autism can be achieved in general education, inclusive environments (Kurth and Mastergeorge, 2010). If only general educators were adequately prepared to address the students' needs (Teffs and Whitbread, 2009).

Famed researchers Simpson and Myles (2008) confirmed in their work with children on the autism spectrum the advantages teachers gain when they learn and acquire skills and experiences to effectively collaborate with the many professionals and family members who team together to provide the child with autism the best educational programming possible. "Significant gains can and do occur when teachers of students with autism possess the necessary depth and breadth in the knowledge and skills needed to provide appropriate instruction" (p. 18).

The ERIC (2011), a highly respected association of educators, administrators and other public officials in the U.S.A., has designed and distributed an outline that encourages improvements to current methods of standard, teacher preparation pedagogy demonstrated at colleges and universities throughout the country. Their guidelines, applicable to all teaching assignments, appear below.

Design principles for clinically based preparation:

- The focus: student learning

- Clinical preparation: integrated throughout every facet of teacher education

- Teacher preparation programs: continually assessed

- Preservice teachers are to be expert in content and how to teach it; they are innovators, collaborators and problem-solvers 


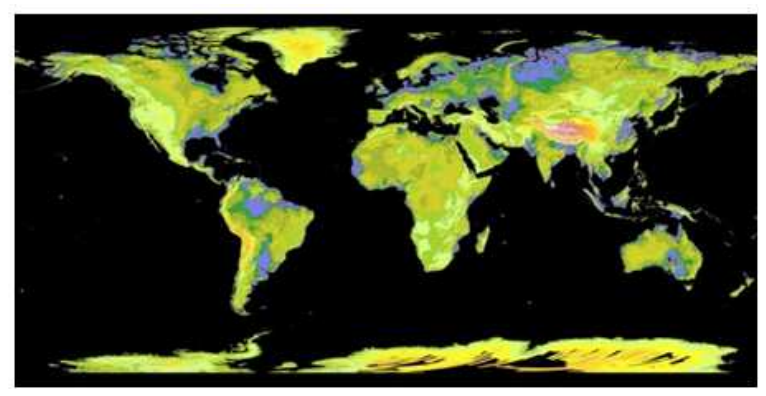

Fig. 2: http://geology.com/nasa/world-topographic-map/

- Interactive professional community: best learning environment for preservice teachers

- Higher education and classroom-based, clinical educators and coaches lead the next generation of teachers

- Throughout their preparation and induction. (They must themselves be effective practitioners, skilled in

- differentiating instruction, proficient in using assessment to monitor learning and provide feedback, persistent

- Searchers for data to guide and adjust practice and exhibitors of the clinical skills.)

- Embedded clinical preparation takes place at specified sites

- Technology applications foster high-impact preparation

- Gathering and using data supports continuous improvement in teacher preparation

- Strategic partnerships: imperative for powerful clinical preparation

Incorporating the above values with research that purports teachers' flexible attitudes and dispositions as the mechanism that increases their instructional effectiveness, expert teachers of inclusive classrooms tailor their instructional style to meet demands of each student and each learning goal of topics presented in the academic venue. Positive student learning outcomes are achieved by teachers who truly feel and demonstrate that they can help students learn. Gaining confidence to provide adequate instruction in the inclusive classroom for students with autism entails not only keen awareness of the students' individual needs, but also encouraging their communication by using pragmatic language; providing literal interpretation and conversation; applying positive behavioral support strategies; and incorporating social skills instruction and practice. Such skills help students with autism make and keep friends, "read" and interpret social situations, better understand body language and proximity and assemble accurate judgments of specific people-to-people interactions (Mehring and Dow, 2001). Over time and with continued professional development that incorporates the latest, researchbased, best practice techniques and information, teachers indeed learn pedagogy and collaborative methods that effectively provide for students with autism the skills to actively achieve and progress in the inclusive classroom setting.

\section{CONCLUSION}

Countries throughout the world (Fig. 2) scramble to learn from specialists of varied global industries and practices the researched and proven-successful information and productive therapies meaningful to students with autism and their families. The prevalence worldwide of individuals with the autism diagnosis is astonishingly high. Researchers continue to probe and experiment to learn more about the condition's etiology, its impact on families and on national economies, methods to accommodate demonstrated behavioral concerns and approaches that can best inform the public and teachers who assist individuals affected by autism. Critically necessary for educators to learn, understand, practice and share with others, are interventions that enable individuals with autism to best cope, thrive and excel in environments that are safe and accepting of diversity. Continued international research and creative programs designed to nurture and encourage children to achieve their potential, can contribute to and benefit greatly individuals with autism and their appreciative families.

\section{REFERENCES}

Autism Speaks, 2008. Autism speaks launches unprecedented global autism public health initiative. Autism Speaks New York.

Bellini, S., D. Henry and C. Pratt, 2011. From intuition to data: Using logic models to measure professional development outcomes for educators working with students on the autism spectrum. Teacher Educ.: J. Teacher Educ. Division Council Exceptional Children, 34: 37-51. DOI: 10.1177/0888406410384153

Centers for Disease Control and Prevention, 2011. Facts about ASDs. Children assessed by the ASSQ (Autism Spectrum Screening Questionnaire). J. Child Psych. Psychiatry Allied Disciplines, 4: 167-75.

Cohen, D.J., 1997. Handbook of Autism and Pervasive Developmental Disorders. 2nd Edn., John Wiley and Sons, New York, ISBN-10: 0471532428, pp: 1092. 
ERIC, 2011. Transforming teacher education through clinical practice: A national strategy to prepare effective teachers. Educ. Digest: Essential Read. Condensed Quick Rev., 76: 9-13.

Kurth, J.A. and A.M. Mastergeorge, 2010. Academic and cognitive profiles of students with autism: Implications for classroom practice and placement. Int. J. Special Educ., 25: 8-14.

Magnusson, P. and E. Saemunsen, 2001. Prevalence of autism in Iceland. J. Autism Dev. Disorders, 31: 153-63. DOI: 10.1023/A:1010795014548

Marchant, P., A. Hussain and K. Hall, 2006. Autistic spectrum disorders and Asian children. Br. J. Educ. Stud., 54: 230-244. DOI:10.1111/j.14678527.2006.00342.x

Mehring, T.A. and M.J. Dow, 2001. Preparing future teachers for students with autistic spectrum disorders. Adv. Special Educ., 14: 69-88. DOI: 10.1016/S0270-4013(01)80008-8

National Autistic Society, 2006. ASD Prevalence. National Autistic Society, London, England.

Posserud, M., A.J. Lundervold, S.A. Lie and C. Gillberg, 2006. The prevalence of autism spectrum disorders: Impact of diagnostic instrument and non-response bias. Soc. Psychiatry Psychiatric Epidemiol., 45: 319-327. DOI: 10.1007/s00127-009-0087-4
Posserud, M., A.J. Lundervold, S.A. Lie and C. Gillberg, 2010. The prevalence of autism spectrum disorders: Impact of diagnostic instrument and non-response bias. Soc. Psychiatry Psychiatric Epidemiol., 45: 319-327. DOI: 10.1007/s00127009-0087-4

Simonoff, E., A. Pickles, T. Charman, S. Chandler and T. Loucas et al., 2008. Psychiatric disorders in children with Autism spectrum disorders: Prevalence, comorbidity and associated factors in a population derived sample. J. Am. Acad. Child Adolescent Psychiatry, 47: 921-929. DOI: 10.1097/CHI.0b013e318179964f

Simpson, R.L. and B.S. Myles, 2008. Educating Children and Youth with Autism: Strategies for Effective Practice. 2nd Edn., PRO-ED, Inc., Austin, ISBN-10: 9781416402107, pp: 537.

Teffs, E.E. and K.M. Whitbread, 2009. Level of preparation of general education teachers to include students with autism spectrum disorders. Curr. Issues Educ., 12: 1-23.

Wong, V.C.N., 2007. Epidemiological study of autism spectrum disorder in China. J. Child Neurol., 23: 67-72. DOI: $10.1177 / 0883073807308702$ 